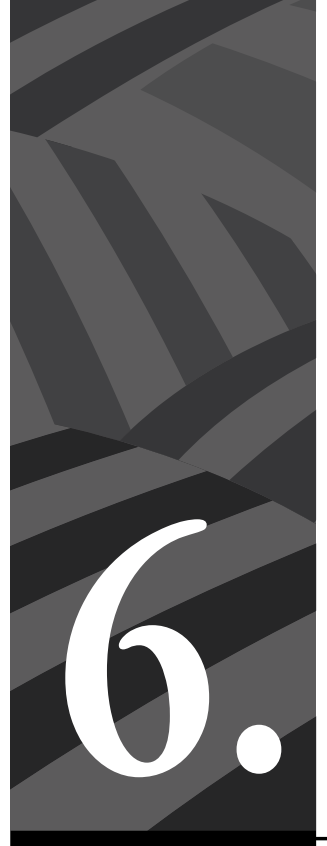

Empleo, educación y desigualdad: las juventudes mexicanas como población vulnerable en tiempos de COVID-19 


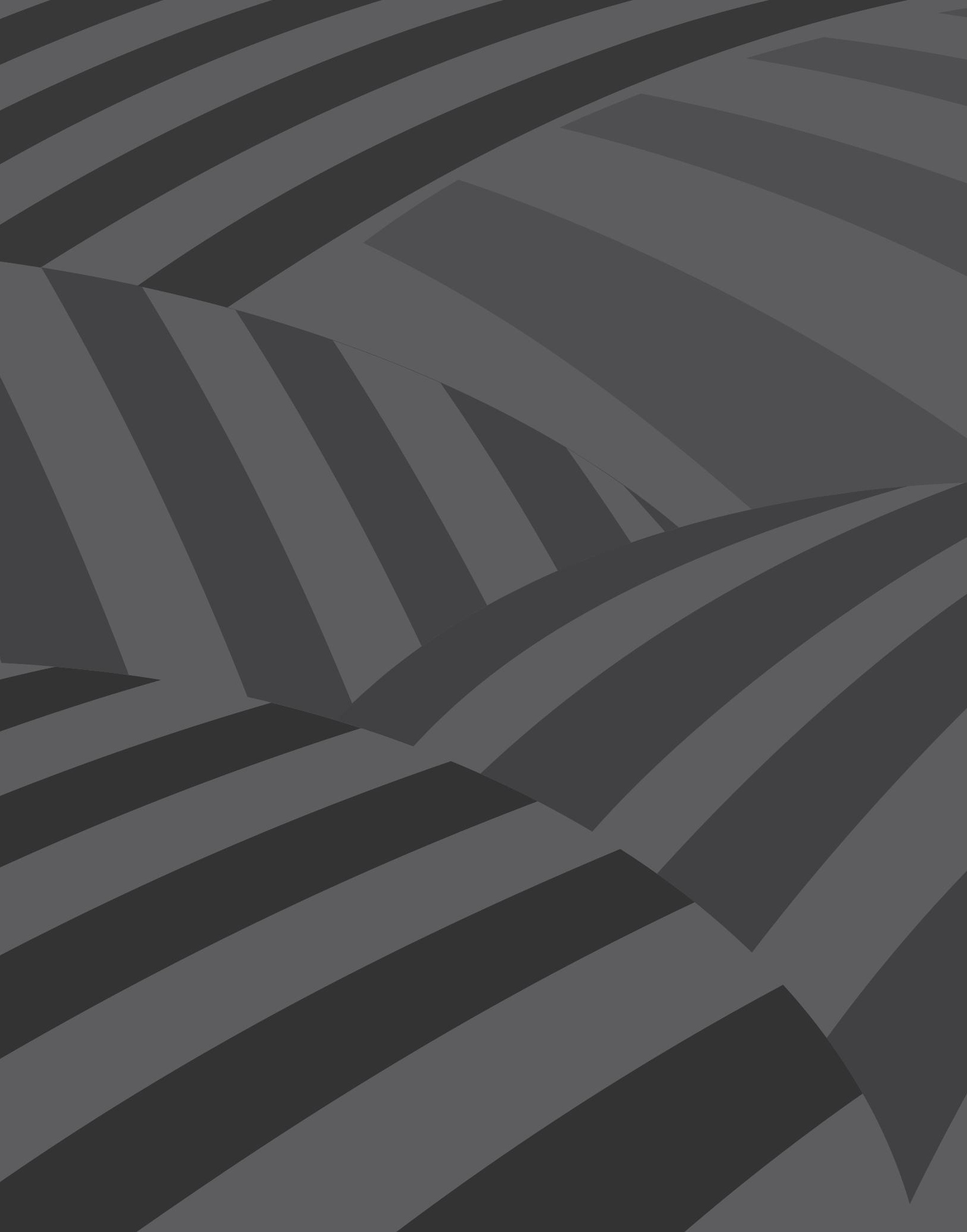




\title{
Empleo, educación y desigualdad: las juventudes mexicanas como población vulnerable en tiempos de COVID-19
}

\author{
Por Ernesto Israel Santillán-Anguiano*, Emilia Cristina González-Machado**
}

Resumen: El presente trabajo reporta las condiciones estructurales de jóvenes mexicanos, como factores que exacerban la precariedad y las asimetrías para hacer frente a las consecuencias y los retos provocados por la alerta sanitaria de la pandemia de COVID-19 declarada por la Organización Mundial de la Salud el 11 de marzo del año 2020. Desde una metodología de análisis documental, se muestran aspectos de las condiciones laborales, educativas y de acceso a la tecnología de información de la población joven. Entre los resultados, se enuncian las dimensiones sociales y económicas que ponen en evidencia la carencia del Estado para atender a esta población, por lo que se mantienen y reproducen las desigualdades simbólicas y materiales lo que pone de manifiesto las limitadas oportunidades de bienestar de las juventudes en México.

Palabras clave: desempleo juvenil, desigualdad social, disparidad tecnológica, derecho a la educación, jóvenes desfavorecidos.

\section{Employment, education and inequality: Mexican youth as a vulnerable population in times of COVID-19}

Abstract: This work reports the structural conditions of young Mexicans, as factors that exacerbate the precariousness and asymmetries to face the consequences and challenges caused by the health alert of the COVID-19 pandemic declared by the World Health Organization on March 11, 2020. A documentary analysis methodology is used, aspects of the working conditions, education and

* Licenciado en Psicología y Maestro en Ciencias Sociales por la por la Universidad Autónoma de Baja California. Doctor en Ciencias y Humanidades para el Desarrollo Interdisciplinario por la Universidad Autónoma de Coahuila. Profesor-Investigador de la Facultad de Pedagogía e Innovación Educativa en la Universidad Autónoma de Baja California. Correo electrónico: santillan_er@uabc.edu.mx

* Licenciada en Psicóloga y Maestra en Ciencias Sociales por la Universidad Autónoma de Baja California. Doctora en Ciencias de la Educación por la Universidad Autónoma de Coahuila. Profesora-Investigadora de la Facultad de Ciencias Humanas en la Universidad Autónoma de Baja California. Correo electrónico: cristina. gonzalez@uabc.edu.mx 
access to information technology of the young population are shown. The results show the social and economic dimensions that highlight the lack of the State to serve this population. In this way, symbolic and material inequalities are maintained and reproduced, which shows the limited opportunities for well-being of young Mexicans.

Keywords: disadvantaged youth, right to education, social inequality, technological gap, youth unemployment

Cómo citar este artículo: González-Machado, Emilia Cristina; Santillán-Anguiano, Ernesto Israel. (2021). Empleo, educación y desigualdad: las juventudes mexicanas como población vulnerable en tiempos de COVID-19. Revista Controversia, 216, 203-227.

Fecha de recepción: 9 de septiembre de 2020.

Fecha de aprobación: 22 de octubre de 2020.

\section{Introducción}

G

nuevo coronavirus denominado por la Organización Mundial de la Salud como SARS-CoV-2, ha evolucionado de una forma rápida a nivel mundial desde su aparición en diciembre del 2019 en la ciudad de Wuhan en China. La forma peculiar como se ha comportado esta epidemia difiere de otras como el Síndrome Respiratorio Agudo Severo o SARS-CoV, que aconteció entre 2002-2003, así como el Síndrome Respiratorio de Oriente Medio o MERS-CoV, el cual tuvo su principal foco en la península arábiga en 2013 (Ramos, 2020). Los coronavirus encuentran una distribución amplia entre aves y mamíferos, incluidos los humanos, y son precursores de enfermedades respiratorias, entéricas, hepáticas y neurológicas. Su denominación proviene de su peculiar apariencia bajo el microscopio, la cual es semejante a una corona. Se conoce que todas las variantes de coronavirus humanos mantienen un ancestro común que probablemente utiliza como agente intermedio a otros animales, pues tienen la capacidad de ser transmisibles entre especies (Aragón, Vargas y Miranda, 2020). El SARS-CoV-2 es el séptimo coronavirus con posibilidades de infectar a la especie humana, a la enfermedad resultante se le denomina COVID-19 (Oliva, 2020). 
En el caso particular de los humanos, el SARS-CoV-2 puede infectar a individuos en diversos rangos de edad. Sin embargo, existe alta incidencia en aquellas personas que presentan condiciones médicas preexistentes, como asma, diabetes y afecciones cardiacas. La mortalidad por COVID-19 se incrementa en personas mayores a los 70 años y al parecer existe una mayor posibilidad de infección en hombres que en mujeres (Palacios, Santos, Velázquez y León, 2020). Aun cuando la atención sanitaria se ha centrado en las personas con factores médicos asociados, la posibilidad de infección rápida también afecta a la población juvenil, la cual tiende a presentar manifestaciones atípicas y de intensidad menor (Huang, Xiuwen, Xinyue, Zhijan, Lingli, Jingjing, Peipie, Yuanhong, Chengyuan y Aman, 2020). La vulnerabilidad de la población joven se incrementa durante los periodos de pandemia, ya que aun cuando no es la población en el rango etario más afectado durante una enfermedad infecciosa, sí mantiene una actividad de propagación en los hogares y en las comunidades (Espada, Orgilés, Piqueras y Morales, 2020).

Las consecuencias en materia de salud, empleo y educación a raíz de la contingencia por el COVID-19 afectan principalmente a países emergentes, con mayor impacto en la población con alto rezago social. Ante este escenario, la pandemia también genera una serie de afectaciones socioeconómicas en la población más pobre y vulnerable. El presente estudio enfoca el análisis en la población joven de México. La pérdida de ingresos, los despidos y las reducciones salariales son elementos propicios para generar condiciones de precariedad laboral, condiciones que prexistentes antes de la contingencia y que se verán exacerbadas por esta. México es uno de los países latinoamericanos donde las proyecciones sugieren un aumento tanto en la pobreza como en la pobreza extrema, con un efecto adicional debido a la disminución de las remesas provenientes de las familias en el extranjero (CEPAL, 2020b). Se espera, por tanto, que ante la precarización económica, la juventud en condiciones de pobreza sea uno de los grupos sociales afectados de manera directa durante los períodos pandémico y postpandémico. En el caso específico de México la población jóvenes es de 37.5 millones 
de personas en el grupo de los 12 y 29 años de edad, que representa un $31.4 \%$ de la población total, de los cuales 3.9 millones sobreviven en condiciones de pobreza, 27 millones mantienen ingresos menores a la línea de bienestar, y 5.4 millones no tienen acceso a la educación ni al empleo (Instituto Mexicano de la Juventud, 2019).

El presente trabajo tiene como base el análisis documental de distintos informes de organismos que abordan aspectos económicos y condiciones laborales, a partir de las primeras aproximaciones ante la pandemia producida por el SARS-CoV-2. El análisis documental como método, busca extraer información de documentos mediante un proceso analítico-sintético, que posibilite recuperar información relevante con el objetivo de generar o presentarla de un modo distinto (Pinto, 1989). Se hace referencia a dos fuentes básicas: La Organización Internacional del Trabajo y la Comisión Económica para América Latina y El Caribe (CEPAL). En particular, la CEPAL ha mantenido un observatorio especializado en el impacto del COVID-19 en América Latina en distintas áreas. Por su parte, la OIT provee informes de seguimiento a la incorporación de la población joven al mercado laboral ante las Naciones Unidas.

\section{Exclusión y juventud}

Etimológicamente la palabra excluir proviene del latín Excludere, constituida a partir del prefijo ex (desde, desplazamiento del interior al exterior) y del verbo claudere (confinar, aislar); por lo que en términos generales cuando se enuncia una acción de exclusión, lo que se intenta describir es el acto de rechazo o de expulsión de una persona de algún espacio o lugar. Para Le Blanc (2020) el acto de negar el derecho de acceso, convierte al sujeto excluido en incompatible con el resto del grupo, generando una frontera que divide a quienes se encuentran dentro o fuera de ella. Cuando se aborda la exclusión social desde la academia, es importante integrar una visión que pueda diferenciar entre aquellos procesos de desventajas sociales, económicas, políticas que afectan la relación entre los individuos y la sociedad. El concepto de exclusión 
social es frecuentemente referido para caracterizar a grupos de la sociedad que experimentan condiciones de privación (Castro, García y López, 2020).

De hecho, a partir de la década de los ochenta del siglo XX, la exclusión y la desigualdad se analizan desde el enfoque de pobreza que orienta el Banco Mundial, el cual tiene como base la teoría de las necesidades básicas que guía la discusión sobre la pobreza en Latinoamérica (Pérez, 2019). Dicho enfoque alude a la capacidad racional de los individuos para administrar sus recursos al momento de satisfacer sus necesidades. Por lo tanto, implica el criterio de que determinado ingreso posibilita el acceso a ciertos recursos y bienes que garanticen algún nivel de bienestar (Cruz-Martínez, 2015).

En los últimos años, los estudios en ciencias sociales asociados a la población joven en América Latina se han focalizado en los procesos de desigualdad cobrando importancia aquellos que se encuentran relacionados a los Derechos Humanos. Una visión que intente clarificar las realidades juveniles latinoamericanas implica entender a la juventud como sujetos sociales e históricos. Si bien la perspectiva basada en los derechos humanos ha permitido la atención de la población joven bajo categorías de "vulnerabilidad”, “discriminación” o "igualdad”, también ha sustentado una visión paternalista o tutelar desde las políticas públicas (Cruz, 2018).

En Latinoamérica las políticas públicas enfocadas en la juventud han mantenido como ejes centrales en las últimas décadas la ampliación de los derechos y el reconocimiento de las diversidades sociales. Las políticas públicas se encuentran normadas a partir de la visión que las sociedades mantienen respecto a la juventud. Vommaro (2016) reconoce cuatro paradigmas donde imperan distintas aproximaciones a lo juvenil: el de moratoria, el de riesgo, el de ciudadanía y el de actores sociales. En la Tabla 1 se presentan de manera esquemática las principales características de cada uno de ellos. 


\section{Tabla 1. Paradigmas asociados a las políticas de juventud en Latinoamérica.}

\begin{tabular}{c|c|c|c|c} 
Paradigma & $\begin{array}{c}\text { Visión de } \\
\text { la juventud }\end{array}$ & $\begin{array}{c}\text { Políticas } \\
\text { públicas }\end{array}$ & Trayectoria & Perspectiva \\
\hline Moratoria & $\begin{array}{c}\text { Proceso de } \\
\text { transición }\end{array}$ & $\begin{array}{c}\text { Universales e } \\
\text { indiferenciadas }\end{array}$ & $\begin{array}{c}\text { Educación para } \\
\text { la vida adulta } \\
\text { y productiva }\end{array}$ & Asistencial \\
\hline Riesgo & $\begin{array}{c}\text { Problema/ } \\
\text { riesgo } \\
\text { Víctima/ } \\
\text { victimario }\end{array}$ & $\begin{array}{c}\text { Compensatorias } \\
\text { Focalizadas }\end{array}$ & $\begin{array}{c}\text { Salud y Sistema } \\
\text { Judicial }\end{array}$ & Asistencial \\
\hline Ciudadanía & $\begin{array}{c}\text { Sujetos de } \\
\text { derecho }\end{array}$ & $\begin{array}{c}\text { Intersectoriales } \\
\text { Incluyentes }\end{array}$ & $\begin{array}{c}\text { Participativos } \\
\text { con búsqueda } \\
\text { de alianzas }\end{array}$ & Participación \\
activa
\end{tabular}

Fuente: Elaboración propia a partir de Vommaro, 2016.

Estos paradigmas siguen presentes en distintas legislaciones latinoamericanas, coexistiendo en una realidad contradictoria, que complejiza la relación de los Estados con los sectores juveniles (Vommaro, 2016). Es decir, puede argumentarse desde el punto de vista exclusivo de las desigualdades, que estas se presentan en América Latina como paradojas o contradicciones, no carentes de tensiones; en las dos últimas décadas se produjo en la región un crecimiento económico a la par de una profundización de la desigualdad al interior de los países. Particularmente, existen grupos sociales que presentan mayor vulnerabilidad y cuyos indicadores son menores, entre ellos se encuentran: las mujeres, los jóvenes y los grupos étnicos indígenas y afrodescendientes (Vommaro, 2019). 
El COVID-19 como enfermedad pandémica ha evidenciado las carencias y desigualdades en los espacios laborales, especialmente en el caso de las mujeres, de jóvenes y personas con trabajos informales. Para la Organización Internacional del Trabajo (OIT), si no se desarrolla una estrategia para mejorar la calidad de las condiciones laborales de los grupos más vulnerables, la recuperación económica, más que ayudar, agravaría las condiciones de injusticia social (OIT, 2020).

\section{Jóvenes y el espejismo del empleo decente}

Antes de la crisis por COVID-19, la OIT ha manifestado la importancia de atender las necesidades de empleo juvenil como uno de los principales desafíos globales. No solo en el marco de la búsqueda de mayor cantidad de empleos, sino en la integración de mejores condiciones. Las y los jóvenes como sector particular, mantienen a nivel internacional un acceso limitado a las oportunidades laborales productivas, salarios decentes y seguridad. Ante la baja calidad del acceso al empleo de jóvenes se corre el riesgo de perder una generación, a la que la economía internacional tendrá que hacer frente (OIT, 2018). Desde el 2016, Naciones Unidas, por medio de la OIT, generó la Iniciativa Global sobre Empleo Decente para los Jóvenes, como un intento para promover el trabajo de esta población a nivel internacional. El objetivo central es apoyar a los distintos Estados Miembros en lograr el cumplimiento de la Agenda 2030. Esta propuesta parte del diagnóstico que expone que, a nivel mundial, 70 millones de jóvenes se encuentran en busca de empleo; 160 millones tienen empleo, no obstante, viven en condiciones de pobreza; cuatro de cada diez jóvenes económicamente activos se encuentran desempleados o son trabajadores pobres, con una tasa de desempleo tres veces mayor que la población adulta (OIT, 2017).

El concepto de trabajo decente fue acuñado desde la 87 reunión de la OIT (1999) y es definido como el trabajo desarrollado en condiciones de libertad, equidad, seguridad y dignidad humana. El trabajo decente busca que converjan la promoción de los derechos laborales, el empleo, 
la protección social y el diálogo social. En el caso específico de México, la protección social se sustenta en un sistema dividido, en el que la posibilidad de acceso se encuentra asociada al tipo de empleo. Por otro lado, la protección de riesgos laborales y los servicios se encuentran fuertemente estratificadas. En términos generales, las personas que mantienen ingresos atípicos o temporales, no cuentan con seguridad social. A partir de la reforma al sistema de pensiones en 1997, el único sistema con el que cuenta la mayoría de la población juvenil se encuentra fundamentado en la capitalización individual. La perspectiva en la vida cotidiana para este grupo etario se encuentra asociada a un alto riesgo de fallecer de causas evitables, mínimo acceso a la educación y al empleo de calidad (Schwarzer y Gómez, 2018).

Ahora, a la pandemia global provocada por el COVID-19 se suma una crisis sanitaria, económica y humana cuya principal consecuencia es el colapso del sistema económico. Las estrategias que normalmente ha utilizado el mercado para salir avante de las crisis previas, parece ser que son insuficientes para mantener las actividades de producción y el encogimiento de la demanda (CEPAL, 2020a). En las condiciones actuales, distintos sectores económicos tienen que hacer frente a pérdidas que ponen en riesgo su funcionamiento. De manera especial y más crítica se identifica a las empresas pequeñas, por lo que las y los trabajadores se encuentran en incertidumbre de despedidos y obtención de ingresos. En una condición aún más grave están quienes realizan trabajos informales, quienes no pueden acceder a ningún tipo de protección. Por lo que, si esta situación es complicada en los países desarrollados, los de ingreso medio y bajo cuentan con un gran número de población que depende de trabajos informales y esta condición limita el acceso a servicios de salud, lo que implica el riesgo de reponerse económicamente para evitar ingresar en la pobreza (OIT, 2020b).

La afectación por el COVID-19 en el mercado laboral no se desarrolla de forma coincidente, existen sectores particulares donde la crisis influye en mayor medida en la actividad económica. En la Tabla 2 se muestran 
los sectores económicos asociados al nivel de riesgo laboral a partir de la crisis por COVID-19.

\section{Tabla 2. Situación mundial de riesgo laboral por sector económico a partir de la crisis por COVID-19.}

\begin{tabular}{l|c|c}
\hline \multicolumn{1}{c|}{ Sector de la economía } & $\begin{array}{c}\text { Nivel de } \\
\text { riesgo }\end{array}$ & $\begin{array}{c}\text { Porcentaje de } \\
\text { participación en } \\
\text { el empleo mundial }\end{array}$ \\
\hline Enseñanza & Bajo & 5.3 \\
\hline Actividades sanitarias y de servicios sociales & Bajo & 4.1 \\
\hline $\begin{array}{l}\text { Administración pública y defensa; seguridad } \\
\text { social de afiliación obligatoria }\end{array}$ & Bajo & 4.3 \\
\hline Servicios públicos esenciales & Bajo & 0.8 \\
\hline Agricultura, ganadería, silvicultura y pesca & Bajo-Media & 26.5 \\
\hline Construcción & Medio & 7.7 \\
\hline Actividades financieras y de seguros & Medio & 1.6 \\
\hline Minería y canteras & Medio & 0.7 \\
\hline $\begin{array}{l}\text { Artes, entretenimiento y recreación, y otras } \\
\text { actividades de servicios }\end{array}$ & Medio-Alto & 5.4 \\
\hline $\begin{array}{l}\text { Transporte y almacenamiento, e información } \\
\text { y comunicación }\end{array}$ & Medio-Alto & 6.1 \\
\hline $\begin{array}{l}\text { Actividades de alojamiento y de servicio de } \\
\text { comidas }\end{array}$ & Alto & 4.3 \\
\hline $\begin{array}{l}\text { Actividades inmobiliarias; actividades admi- } \\
\text { nistrativas y comerciales }\end{array}$ & Alto & 4.7 \\
\hline \begin{tabular}{l} 
Industrias manufactureras \\
\hline $\begin{array}{l}\text { Comercio al por mayor y al por menor; repa- } \\
\text { ración de vehículos de motor y motocicletas }\end{array}$
\end{tabular} Alto & 13.9 \\
\hline
\end{tabular}

Fuente: Elaboración propia a partir de OIT, 2020b.

A partir de estos datos se puede inferir que los servicios que se ven afectados de manera drástica se asocian a sectores intensivos, es decir, 
aquellos de los cuales dependen millones de personas, como por ejemplo: la agricultura, hotelería, restaurantes y minería; trabajos que requieren de una alta contribución de mano de obra en contraste con la cantidad del capital. Son trabajos comúnmente mal pagados y poco calificados, entre los que se incluyen los servicios de alojamiento, de comida y comercio al por menor y que seguramente serán los más afectados durante el periodo de la pandemia. Cerca del $40 \%$ de la población mundial se encuentra en alta condición de riesgo laboral, y corresponde a trabajos informales (OIT, 2020b).

La informalidad laboral o empleo atípico es una condición específica en la cual un gran número de personas se desenvuelven en el mundo. El empleo informal incorpora un amplio espectro de ocupaciones en una diversidad de espacios laborales y en él se incluyen: comerciantes, comerciantes ambulantes, asalariados informales que no tienen beneficios ni protección social, empleos domésticos y del campo (Temkin y Cruz, 2019). Para Román (2017), existen cuatro elementos a tomar en cuenta en la relación juventud-informalidad laboral en México: 1) el primero está asociado el carácter que ha tenido el trabajo informal como la principal estructura que absorbe la mano de obra; 2) el segundo se asocia al crecimiento sostenido que este sector ha mantenido y que actualmente se encuentra en el $57 \%$ del empleo total a nivel nacional; 3) el tercer factor se relaciona directamente a las afectaciones que el empleo informal tiene sobre la población juvenil, al ser el sector más afectado e imposibilitado en su acceso a condiciones laborales adecuadas; 4) el cuarto factor se orienta especialmente a la incorporación cada vez mayor de jóvenes como mano de obra informal, con su consecuente pérdida del poder adquisitivo y mínimo efecto en las economías regionales y nacional.

Otro elemento sustancial en la relación jóvenes-mercado laboral es la falta de empleo o la desocupación. Durante el 2019 los indicadores del mercado laboral en Latinoamérica fueron negativos, ya que se encuentran asociados a la desocupación y una menor oferta de empleos respecto a los años anteriores. En el caso específico del empleo entre jóvenes de 
15 a 24 años, este decreció en el periodo del 2013 al 2018 en 16 países de América Latina (ver Tabla 3). En comparación con el empleo general donde se desarrollaron valores positivos, se puede afirmar que incluso en periodos de crecimiento económico, existen factores que afectan de manera negativa el acceso al empleo de la población joven (OIT, 2019).

\section{Tabla 3. Variación porcentual del empleo total y el juvenil 2013-2018 en Latinoamérica.}

\begin{tabular}{c|c|c|c|c|c|c|c}
\hline $\begin{array}{c}\text { Variación } \\
\text { porcentual }\end{array}$ & 2013 & 2014 & 2015 & 2016 & 2017 & 2018 & $\begin{array}{c}\text { Promedio } \\
2013-2018\end{array}$ \\
\hline Empleo total & 1.85 & 1.58 & 1.55 & 1,41 & 1.91 & 2.28 & 1.76 \\
\hline Empleo juvenil & -0.83 & 0.10 & -0.72 & -0.07 & -1.05 & -0.20 & -0.46 \\
\hline
\end{tabular}

Fuente: Elaboración propia a partir de datos de OIT, 2019.

Es importante señalar que uno de cada cinco jóvenes entre 15 y 24 años en Latinoamérica se encuentra desempleado, esto implica una tasa tres veces superior a la población mayor a 25 años (OIT, 2019). En el caso de México, la población juvenil desempleada entre 14 y 29 años, es de $6.3 \%$ respecto al $2.5 \%$ de la población adulta (Hernández, 2020).

\section{Jóvenes: La educación fracturada}

La educación se ha convertido desde el siglo XIX en uno de los principales elementos asociados a los derechos fundamentales de los seres humanos. El siglo XX se caracterizó por incorporar de manera masiva la educación en las sociedades por medio de la escolarización. De esta forma, el acceso a la educación terminó transformándose en la garantía por medio de la cual los Estados aseguraban un mínimo de instrucción obligatoria (Acosta, 2017). Desde el surgimiento de la Declaración Universal de los Derechos Humanos en 1948, una serie de referentes internacionales arroparon a la educación dentro del gran abanico de los derechos humanos, intentando generalizar su gratuidad y obligatoriedad. 
La Convención Relativa a la Lucha contra las Discriminaciones en la Esfera de la Enseñanza en 1960, asume la responsabilidad del Estado y la igualdad de acceso y oportunidades. El Pacto Internacional de Derechos Económicos, Sociales y Culturales y el Pacto Internacional de Derechos Civiles y Políticos, ambos declarados en 1966, enuncian el acceso gratuito y refuerzan la obligatoriedad de los Estados, por lo que brindan un referente en el respeto de las minorías étnicas y culturales para acceder a los mismo servicios que el resto de la población. La Conferencia Mundial sobre Educación para Todos en 1990, definió las estrategias para el desarrollo de políticas públicas en materia de educación básica. Finalmente, la Declaración de Incheon para la Educación 2030 y la Agenda 2030 sobre el Desarrollo Sostenible, ambas en 2015, intentan orientar y fortalecer la perspectiva global sobre el derecho a la educación para todos los seres humanos (González-Machado y Santillán-Anguiano, 2018).

Lo que en la actualidad se asume como escolarización, es el derecho de acceso y permanencia dentro de los sistemas educativos. Es un hecho que las generaciones recientes de jóvenes en América Latina han tenido un mayor ingreso a la educación que sus antecesoras. Dentro del marco de la Agenda 2030 y el logro de los Objetivos de Desarrollo Sostenible, se busca alcanzar la conclusión de la educación secundaria en todos los sectores, así como la igualdad de oportunidades de aprendizaje para ingresar más adelante a los estudios universitarios. Se puede afirmar que de manera oficial existe una preocupación mundial por incentivar tanto las políticas educativas, como la formación escolar de los sectores juveniles de las sociedades. Se espera que la mayoría de la población logre terminar el nivel medio, aun cuando en Latinoamérica desde el 2010 ha decrecido la escolarización, en un $2.5 \%$ en niñas y niños entre 9 y 11 años; un $21 \%$ entre los que tienen 15 y 17 años; un $36.5 \%$ en el rango de 21 a 23 años y un $46.1 \%$ en los de 31 a 33 años; siendo estos porcentajes mayores en la población campesina. Dadas las condiciones actuales en las que el desempleo aumenta, las oportunidades de acceso laboral disminuyen, y cada vez ingresan mayor cantidad de 
jóvenes al trabajo informal, se establecen las condiciones para que en América Latina cerca de un $22 \%$ de la juventud no estudie ni trabaje (D’Alessandre, 2017; López, Opertti y Vargas, 2017 y OIT, 2019).

Es posible que los jóvenes de familias económicamente más vulnerables se vean en la necesidad de incorporarse al espacio laboral. En el caso de las y los jóvenes, pudiera existir una elevada carga de trabajo doméstico, así como mayores restricciones para poder reincorporarse a las escuelas y a trabajos necesarios para apoyar la economía familiar. Esto es especialmente grave en los países donde el desempleo juvenil mantiene tasas superiores al promedio regional (CEPAL, 2020c). Esta afectación puede mantener un elevado impacto territorial, pues las regiones más afectadas serán aquellas que mantienen concentraciones de población más pobre o que se encuentran establecidas en regiones de bajo desarrollo económico. En estos espacios socialmente más desiguales, la posibilidad de dar respuesta sanitaria oportuna disminuye, por lo que los impactos y afectaciones serán mayores en los sectores con vulnerabilidad urbana para continuar afectando en los espacios rurales (CEPAL, 2020d).

En relación con el vínculo entre la educación y el trabajo informal, existe una asociación directa entre el término de los estudios de bachillerato como elemento facilitador para el acceso a empleos formales. En América Latina, la imposibilidad de continuar los estudios, impide el desarrollo de capacidades que finalmente tendrán una repercusión productiva en las juventudes. Esta pérdida relacionada con el detrimento de niveles educativos básicos, identifica problemáticas de mayor profundidad en los sistemas educativos de la región (CEPAL, 2018). En el caso de México existe un considerable rezago en comparación con muchos de los países de la OCDE, especialmente son afectados los grupos de niños y jóvenes que provienen de condiciones socioeconómicas de mayor vulnerabilidad, pues estructuralmente se mantienen las disparidades sociales (Márquez, 2017). 
Los centros escolares de los países latinoamericanos han tenido que cerrar a partir de la pandemia, lo que ha afectado a más de 115 millones de estudiantes de todos los niveles (CEPAL, 2020c). En el caso específico de México la pandemia ha afectado durante el ciclo 2019-2020 el cierre de 258 mil escuelas, 36.6 millones de estudiantes y a 2.1 millones de docentes (Secretaría de Educación Pública, 2019). Los países de la región han generado estrategias para continuar con la enseñanza a partir de la educación a distancia. Sin embargo, las brechas educativas entre los sistemas públicos y privados han golpeado especialmente a las capas más pobres. El acceso restringido o inexistente a herramientas tecnológicas y al Internet, las condiciones de hacinamiento y la presencia en los hogares de adultos con incipientes niveles de educación, generan condiciones propicias para una elevada probabilidad de abandono escolar, principalmente en aquellos escenarios con pérdida de ingresos (CEPAL, 2020c). En el caso de la brecha digital, México se encuentra ubicado en el lugar 87 a nivel internacional, y en octavo lugar de los países latinoamericanos en acceso a las Tecnologías de la Información (Lloyd, 2020).

En un análisis de la Encuesta Nacional sobre Disponibilidad y uso de Tecnologías de la Información en los Hogares 2019, Itzkuauhtli Zamora encuentra que el acceso al servicio de internet mantiene una distribución desigual en términos territoriales. En México, el 65.5\% de los habitantes tiene acceso a Internet en las ciudades, en comparación con un 23.4\% que lo tiene en espacios rurales (Zamora, 2020). En el registro de hogares que tienen acceso a Internet y cuentan con equipos de cómputo para poder sobrellevar el trabajo en casa o el seguimiento escolar a distancia, se puede observar una tendencia a preferir la conexión a la red que adquirir equipo de cómputo. En la Tabla 4, puede observarse la relación en el incremento/decremento en conectividad a Internet y equipamiento de cómputo y cómo, a partir del 2017, se invirtió la prioridad de acceso sobre la de equipo. 


\section{Tabla 4. Porcentaje de hogares equipados en México con equipo de cómputo e Internet}

\begin{tabular}{|c|c|c|c|c|c|c|c|c|c|c|}
\hline Acceso & 2010 & 2011 & 2012 & 2013 & 2014 & 2015 & 2016 & 2017 & 2018 & 2019 \\
\hline Computadora & 29.8 & 30.0 & 32.2 & 35.8 & 38.3 & 44.9 & 47.0 & 50.9 & 44.9 & 44.3 \\
\hline Internet & 22.2 & 23.3 & 26.0 & 30.7 & 34.4 & 39.2 & 45.6 & 45.4 & 52.9 & 56.4 \\
\hline
\end{tabular}

Fuente: Elaboración propia con datos de Zamora, 2020.

En el mismo estudio se reporta que un $45 \%$ de la población de estrato socioeconómico bajo cuenta con acceso a Internet en sus casas, en contraste con el $92 \%$ del estrato más alto. Seis de cada diez de los hogares más pobres que tienen equipo de cómputo, no pueden contratar Internet por motivos económicos y tres de cada diez hogares pobres se encuentran en espacios geográficos que no cuentan con el servicio. En la Tabla 5 se muestra la relación de las causas asociadas a la falta de acceso a Internet por estrato socioeconómico, en ella puede observarse cómo existe una relación directa entre las condiciones sociales y el acceso a Internet por causas estructurales.

\section{Tabla 5. Causas para no acceder a Internet por estrato socioeconómico.}

\begin{tabular}{c|c|c|c}
\hline $\begin{array}{c}\text { Estrato } \\
\text { socioeconómico }\end{array}$ & $\begin{array}{c}\text { Falta de recursos } \\
\text { económicos }\end{array}$ & $\begin{array}{c}\text { No les interesa } \\
\text { o no necesitan }\end{array}$ & $\begin{array}{c}\text { No existe servicio } \\
\text { en localidad }\end{array}$ \\
\hline Bajo & 60.2 & 10.1 & 26.1 \\
\hline Medio Bajo & 47.2 & 28.2 & 15.3 \\
\hline Medio Alto & 50.9 & 34.6 & 2.0 \\
\hline Alto & 34.8 & 35.2 & 3.9 \\
\hline
\end{tabular}

Fuente: Elaboración propia con datos de Zamora, 2020. 
Para Ortiz (2017), la base del uso de las TIC en México se encuentra en la posibilidad de acceso a Internet. Los estratos más pobres de la población están conectándose a la red mediante un equipamiento elemental tecnológicamente como es la telefonía celular. Las prácticas de usos de teléfonos celulares en México están monopolizadas en el 89.3\% por las dos compañías. La concentración del mercado en estas empresas descarta la posibilidad de mejoras en la calidad y cobertura. Para Murillo y Duk (2020) la educación a distancia en estas condiciones es una ilusión, y solo una alternativa para quienes tienen acceso a equipos de calidad y conectividad en el hogar. Aquellos estudiantes que no cuentan con las condiciones materiales no pueden ser beneficiarios de esta posibilidad. Existen millones de estudiantes en el continente que no tienen acceso en los hogares a elementos tan básicos como la electricidad.

\section{Discusión y conclusiones}

La crisis sanitaria, económica y social que trae consigo el COVID-19 a nivel mundial ha afectado especialmente a los países emergentes, y se espera que tenga una repercusión negativa en aquellos grupos sociales de mayor vulnerabilidad. Aun cuando la población joven pareciera estar exenta de afectación física en comparación con las personas de la tercera edad, se puede afirmar que el impacto entre jóvenes se enfocará principalmente en las áreas del empleo y la educación.

México mantiene indicadores de empleo informal y desempleo juvenil mayores a otros países de la región. En estas condiciones, la precariedad y la informalidad en el empleo, así como las posibilidades de despido se acentúan al ser la población joven un grupo con mayor vulnerabilidad. Internacionalmente, las y los jóvenes tienen dificultades al momento de acceder a empleos y salarios dignos; particularmente en México que históricamente cuenta con una población mayor en este rango etario, la afectación a largo plazo implica el ingreso de un futuro con adultos en condiciones laborales precarias. La pandemia ha develado en los sectores juveniles una situación de crisis de empleabilidad. 
El cierre de empresas y pequeños negocios, así como todos los centros de trabajo que tradicionalmente contratan mano de obra joven, han dejado sin protección económica y social a una elevada porción de la población económicamente activa. El desempleo juvenil es una de las condiciones extremas que México tiene que resolver, la falta de empleo ya era un elemento sustancial de alerta económica y social antes de la incursión del COVID-19. El desempleo no solo excluye a la juventud de la posibilidad de acceso a una condición económica segura, tiene un efecto directo en la integración al mercado laboral informal.

El caso de la educación tiene aristas más sutiles, pero impactos similares. Si bien puede afirmarse que en las últimas décadas ha aumentado el ingreso de grandes porciones de la población a la educación escolarizada. También existe evidencia de que el acceso a la educación de calidad mantiene fuertes condiciones de desigualdad de clase, género y raza. La desigualdad educativa en México se presenta según los contextos territoriales, ya que en el espacio físico se reflejan las condiciones de disparidad estructural, donde la población pobre indígena y rural se encuentran en circunstancia de desventaja, frente a la población suburbana y urbana. Aun cuando la educación básica tiene una cobertura completa, según los indicadores oficiales, el problema del abandono y la deserción escolar se sitúan precisamente en el último tramo de la educación secundaria y el bachillerato. Es la población juvenil donde converge la renuncia a las posibilidades educativas y el ingreso a la vida laboral o al desempleo. En condiciones pandémicas, el cierre de espacios escolares puede ser un factor predisponente para que una proporción importante de la población joven en condiciones de vulnerabilidad abandone la escuela. La necesidad de conseguir ingresos familiares y el fracaso del sistema educativo para desarrollar estrategias de aprendizajes a distancia son los dos elementos que se suman a la tensión en el interior de los hogares con menos recursos económicos. Son dos factores que configuran la ecuación de la desigualdad educativa juvenil. 
En ese mismo sentido, el equipamiento tecnológico en los hogares mexicanos, es otro factor que se relaciona con la juventud, la educación y la precariedad. Desde los primeros días de la suspensión de las escuelas en México, el discurso oficial se centró en convencer a la población de los beneficios de una estrategia de educación a distancia basada en las tecnologías. Esta visión que asume que los procesos educativos son similares en su dinámica a las estructuras laborales, que apuestan por el teletrabajo en una sociedad aparentemente dominada por las tecnologías, carece en el menor de los casos de una falta de lectura de los datos que convierten a México en uno de los países con rezago en infraestructura de acceso a la red y de equipamiento en los hogares. Esta percepción no toma en cuenta la inhabilitación en términos tecnológicos en los hogares, donde en un mismo espacio y con un mínimo equipo la familia entera tiene que trabajar y educarse. En esta situación, no solo se encuentra la población con acceso a Internet y equipo, sino que estas mismas condiciones son experimentadas por toda la comunidad docente, en cuyos hombros el sistema educativo ha soportado la estrategia de enseñanza en los hogares. Los casos extremos los experimentan las capas más desfavorecidas donde no existe la posibilidad mínima de acceder a equipos especializados de cómputo y mucho menos se tiene la probabilidad de aspirar a los servicios de Internet en el hogar.

Una población juvenil con graves condiciones de desigualdad laboral, con una predisposición estructural para abandonar o no acceder a los siguientes niveles escolares; un equipamiento y conectividad insuficiente, junto con una comunidad docente sin las condiciones de trabajo y capacitación, se enfrentan a una estrategia educativa que apuesta por un proceso de autorregulación, sin considerar un plan de acción que impacte de manera positiva al proceso educativo. Esta pandemia, ha mostrado y ratificado las profundas desigualdades de un sistema económico que no tiene posibilidades ni intenciones de soportar y apoyar a los que menos tienen. 


\section{Referencias:}

Acosta, Felicitas (2017). El derecho a la educación: análisis desde una perspectiva de internacionalización de la escolarización. Academia. Revista sobre enseñanza del derecho de Buenos Aires, 15 (29), 173-195. Recuperado de https://dialnet.unirioja.es/servlet/articulo? codigo $=6073731$

Aragón, Ranferi, Vargas, Ivaán y Miranda, María (2020). COVID-19 por SARSCoV-2: la nueva emergencia de salud. Revista Mexicana de Pediatría, 86 (6), 213-218. Recuperado de https://dx.doi.org/10.35366/9187

Castro, Laura, García, Cirilo. y López, Raúl. (2020). Exclusión social, inclusión política y autoestima de jóvenes en pobreza, Monterrey, México. Revista de Ciencias Sociales, 26 (1), 38-50. Recuperado de https://dialnet.unirioja.es/ servlet/articulo? codigo $=7384404$

Comisión Económica para América Latina y el Caribe (2020a). América Latina y el Caribe ante la pandemia del COVID-19: efectos económicos y sociales. Informe Especial COVID-19 No.1. Ginebra: Comisión Económica para América Latina y el Caribe. https://www.cepal.org/es/publicaciones/45337america-latina-caribe-la-pandemia-COVID-19-efectos-economicos-sociales

Comisión Económica para América Latina y el Caribe (2020b). Informe Especial COVID-19 Número 3. Ginebra: Comisión Económica para América Latina y el Caribe https://repositorio.cepal.org/bitstream/handle/11362/45527/ S2000325_es.pdf?sequence $=5$ \&isAllowed $=y$

Comisión Económica para América Latina y el Caribe (2020c). El desafío social en tiempos del COVID-19. Ginebra: Comisión Económica para América Latina y el Caribe. https://www.cepal.org/es/ publicaciones/45527-desafio-social-tiempos-COVID-19

Comisión Económica para América Latina y el Caribe (2020d). La pandemia por COVID-19 podría incrementar el trabajo infantil en América Latina y el Caribe. Andalucía: Comisión Económica para América Latina y el Caribe. Recuperado de https://repositorio.cepal.org/bitstream/handle/11362/45679/1/ NotaTecnica1OIT-CEPAL_es.pdf

Comisión Económica para América Latina y el Caribe (2018). La ineficiencia de la desigualdad, 2018. Santiago: Comisión Económica para América Latina y el Caribe. Recuperado de https://repositorio.cepal.org/bitstream/ handle/11362/43442/6/S1800059_es.pdf 
Cruz, Mario (2018). Juventud y derechos políticos. Una nueva narrativa de derechos humanos en América Latina. Revista de Direito Brasileira, 19 (8), 250-275. Recuperado de https://indexlaw.org/index.php/rdb/article/ view/4461/3537

Cruz-Martínez, Gibrán (2015). Esfuerzo de bienestar y pobreza desde el enfoque monetarista y de capacidades: Análisis transnacional en América Latina y el Caribe (1990-2010). Política y Sociedad, 52 (3), 631-659. Recuperado de doi: http://dx.doi.org/10.5209/rev_POSO.2015.v52.n3.45521

D’Alessandre, Vanesa (2017). La relación de las y los jóvenes con el sistema educativo ante el nuevo pacto de inclusión en el nivel medio, En Nestor López, Renato Opertti y Carlos Vargas. Adolescentes y jóvenes en realidades cambiantes. Notas para repensar la educación secundaria en América Latina. París: Organización de las Naciones Unidas para la Educación, la Ciencia y la Cultura.

Espada, José, Orgilés, Mireia, Piqueras, José y Morales, Alexandra (2020). Las Buenas Prácticas en la Atención Psicológica Infanto-juvenil ante el COVID-19. Clínica y Salud, 31 (2), 109-113. Recuperado https://doi. org/10.5093/clysa2020a14

González-Machado, Emilia y Santillán-Anguiano, Ernesto (2018). La educación de los jóvenes como derecho humano ante el rezago social en México, en Jesús Trujillo y Laura Dino (Coords.). Debate legislativo y educación. El artículo tercero a cien años de la Constitución Política de 1917. Chihuahua: Red de Investigadores Educativos Chihuahua A. C. Recuperado de: https:// dialnet.unirioja.es/servlet/articulo? codigo $=7040857$

Hernández, Jonthan (2020). Desempleo en México por características sociodemográficas, 2005-2018. Economía UNAM, 17 (50), 166-181. Recuperado de http://revistaeconomia.unam.mx/index.php/ecu/article/view/524

Huang, Lei, Xiuwen, Zhang, Xinyue, Zhang, Zhijan, Wei, Lingli, Zhang, Jingjing, Xu, Peipie, Liang, et al. (2020). Rapid asymptomatic transmission of COVID-19 during the incubation period demonstrating strong infectivity in a cluster of youngsters aged 16-23 years outside Wuhan and characteristics of young patients with COVID-19: A prospective contact-tracing study. Journal of Infection, 8 (6), 1-13. Recuperado de https://doi.org/10.1016/j. jinf.2020.03.006 
Instituto Mexicano de la Juventud (2019). Hacia una perspectiva de juventud. Ciudad de México: Instituto Mexicano de la Juventud. Recuperado de https://www.gob.mx/cms/uploads/attachment/file/484348/Hacia_una_ Perspectiva_de_Juventud.pdf

Le Blanc, Guillaume (2020). Derecho de ciudad. Ciencias Sociales y Educación, 9 (17), 287-309. Recuperado de https://doi.org/10.22395/csye.v9n17a14

Lloyd, Marion (2020). Desigualdades educativas y la brecha digital en tiempos de COVID-19. En Hugo Casanova (Coord.), Educación y pandemia: una visión académica. Ciudad de México: Universidad Nacional Autónoma de México, Instituto de Investigaciones sobre la Universidad y la Educación.

López, Nestor; Opertti, Renato y Vargas, Carlos (2017). Adolescentes y jóvenes en realidades cambiantes. Notas para repensar la educación secundaria en América Latina. Organización de las Naciones Unidas para la Educación, la Ciencia y la Cultura. Recuperado de http://repositorio.minedu.gob.pe/bitstream/handle/MINEDU/5307/Adolescentes \% 20y \% 20 j \% C3 \% B3 venes \% 20en \% 20realidades \% 20cambiantes \% 20notas \% 20 para \% 20repensar \% 20la \% 20educaci \% C3 \% B3n \% 20secundaria \% 20en \% 20 Am \% C3 \% A9rica \% 20Lati.pdf?sequence $=1$

Márquez, Alejandro (2017). A 15 años de PISA: resultados y polémicas. Perfiles Educativos, 39 (156), 3-15. Recuperado de http://www.scielo.org.mx/pdf/ peredu/v39n156/0185-2698-peredu-39-156-00003.pdf

Murillo, Javier y Duk, Cynthia (2020) . El COVID-19 y las Brechas Educativas. Revista Latinoamericana de Educación Inclusiva, 14 (1), 11-13. Recuperado de http://dx.doi.org/10.4067/S0718-73782020000100011

Oliva, José (2020). SARS-COV-2: Origen, estructura, replicación y patogénesis. Alerta. Revista Científica del Instituto Nacional de Salud, 3 (2), 23-30. Recuperado de doi: https://doi.org/10.5377/alerta.v3i2.9619

Organización Internacional del Trabajo (2020). Observatorio de la OIT: La COVID-19 y el mundo del trabajo. Quinta edición. Ginebra: Organización Internacional del Trabajo. Recuperado de https://www.ilo.org/ wcmsp5/groups/public/@dgreports/@dcomm/documents/briefingnote/ wcms_749470.pdf

Organización Internacional del Trabajo (2020b). El COVID-19 y el mundo del trabajo. Segunda edición. Estimaciones actualizadas y análisis. Ginebra: 
Organización Internacional del Trabajo. Recuperado de: https://www.ilo. org/wcmsp5/groups/public/---dgreports/---dcomm/documents/briefingnote/wcms_740981.pdf

Organización Internacional del Trabajo (2019). Panorama Laboral 2019. América Latina y el Caribe. Ginebra: Organización Internacional del Trabajo. Recuperado de https://www.ilo.org/wcmsp5/groups/public/---americas/--ro-lima/documents/publication/wcms_732198.pdf

Organización Internacional del Trabajo (2018). Programa de la OIT sobre empleo juvenil. Ginebra: Organización Internacional del Trabajo. Recuperado de https://www.ilo.org/employment/areas/youth-employment/ WCMS_627866/lang--es/index.htm

Organización Internacional del Trabajo (2017). Empleo Decente para los Jóvenes. La iniciativa global para la acción. Ginebra: Organización Internacional del Trabajo. Recuperado de https://www.ilo.org/global/topics/youthemployment/publications/WCMS_488474/lang--es/index.htm

Organización Internacional del Trabajo (1999). 87a Reunión del Comité de Internacional del Trabajo. Memoria del Director General. Trabajo decente. Organización Internacional del Trabajo. Ginebra: Organización Internacional del Trabajo. Recuperado de https://www.ilo.org/public/spanish/standards/relm/ilc/ilc87/rep-i.htm

Ortíz, Uriel (2017). La reducción de la brecha digital en México: Microtelcos una respuesta (Tesis de Maestría). Recuperada de http:// repositorio-digital.cide.edu/bitstream/handle/11651/2251/158054. pdf? sequence $=1$ \&isAllowed $=\mathrm{y}$

Palacios, Mariali; Santos, Edgar; Velázquez, Manuel y León, Moisés (2020). COVID-19, a worldwide public health emergency. COVID-19, a worldwide public health emergency. Revista Clínica Española, 220 (2), 1-7. Recuperado de https://doi.org/10.1016/j.rce.2020.03.001

Pérez, Juan (2019). Las desigualdades y la re-politización de lo social en América Latina. Encartes, 2 (4), 1-47. Recuperado de https://encartesantropologicos.mx/desigualdad-politizacion-america-latina/

Pinto, María (1989). Introducción al análisis documental y sus niveles: el análisis de contenido. Boletín de la ANABAD, 39 (2), 323-342. 
Ramos, Celso (2020). COVID-19: la nueva enfermedad causada por un coronavirus. Salud Pública de México, 62 (2), 225-227. Recuperado de http:// dx.doi.org/10.21149/11276

Román, Yuliana (2017). Jóvenes y sector informal en el Estado de México. Un grupo en desventaja. Perspectivas Sociales, 19 (2), 41-60. Recuperado de https://dialnet.unirioja.es/servlet/articulo? codigo $=6292201$

Schwarzer, Helmut y Gómez, Martín (2018). Desafíos de la protección social en México. Trabajo,10 (15), 261-283. Recuperado de http://www. relats.org/documentos/FTGeneral.RevistaTrabajoM\%C3\% A9xico2018. pdf\#page $=263$

Secretaría de Educación Pública (2019). Boletín No. 254 Inician el periodo vacacional de fin de año más de 25 millones de alumnos de educación básica. Ciudad de México. Secretaría de Educación Pública. Recuperado de https:// www.gob.mx/sep/articulos/boletin-no-254-inician-el-periodo-vacacionalde-fin-de-ano-mas-de-25-millones-de-alumnos-de-educacion-basicaz

Temkin, Benjamín y Cruz, Jorge (2019). Determinantes subnacionales de la informalidad laboral en México. Realidad, Datos y Espacios. Revista Internacional de Estadística y Geografía, 10 (2), 46-61. Recuperado de https://rde.inegi.org.mx/index.php/2019/08/20/ determinantes-subnacionales-de-la-informalidad-laboral-en-mexico/

Vommaro, Pablo (2019). Desigualdades, derechos y participación juvenil en América Latina: acercamientos desde los procesos generacionales. Revista Direito e Práxis, 10 (2), 1192-1213. Recuperado de https://doi. org/10.1590/2179-8966/2019/40829.

Vommaro, Pablo (2016). Hacia los enfoques generacionales e intergeneracionales: tensiones y perspectivas en las políticas públicas de juventud en América Latina. Revista Latinoamericana de Estudios de Familia, 8, 119135. Recuperado de doi: 10.17151/rlef.2016.8.8.

Zamora, Itzkuauhtli (2020). Accesibilidad y uso de Internet en México. La ENDUTIH a la luz de COVID-19. Visor Ciudadano. (70). 1-27. Recuperado de http://www.bibliodigitalibd.senado.gob.mx/bitstream/handle/123456789/4869/VC_70.pdf? sequence $=1$ \&isAllowed $=y$ 